\title{
Evaluation of visual function in preschool-age children using a vision screening protocol
}

This article was published in the following Dove Press journal:

Clinical Ophthalmology

\section{Tsukasa Satou \\ Yoshiaki Takahashi \\ Misae Ito \\ Hiroshi Mochizuki \\ Takahiro Niida}

Department of Orthoptics and Visual Sciences, School of Health Sciences, International University of Health and Welfare, Otawara, Tochigi, Japan
Correspondence: Tsukasa Satou Department of Orthoptics and Visual Sciences, School of Health Sciences, International University of Health and Welfare, 2600-I Kitakanemaru, Otawara, Tochigi 324-850I, Japan

$\mathrm{Tel}+8 \mathrm{I} 287243642$

Fax +8I 28724363 I

Email tsukasa@iuhw.ac.jp
Purpose: To evaluate the relationship between uncorrected visual acuity and refraction and binocular function using a vision screening protocol.

Methods: In total, 760 children (3-6 years old) who were enrolled in 4 nursery schools in Otawara, Japan, were recruited; a total of 1,520 eyes were examined. We assessed uncorrected near visual acuity, manifest refraction, stereopsis, and eye position. Subjects were divided into 4 subgroups according to the lowest uncorrected near visual acuity value compared between the 2 eyes: group 1 (visual acuity [VA] $\leq 0.00$ [logarithm of the minimum angle of resolution]), group 2 (VA 0.15-0.05), group 3 (VA 0.52-0.22), and group 4 (VA $>0.52$ ). These parameters were compared among the groups.

Results: The sample number of each of the 4 sub-groups was as follows: group 1, 608; group 2, 114; group 3, 27; and group 4, 11. The median spherical equivalent values were -1.13 diopter (D) in group 1 and -1.00 in group 2, which were more myopic than group 4. Median cylindrical power in group 1 was $0.25 \mathrm{D}$, and was the lowest among all groups. In group 1 , median anisometropia was $0.38 \mathrm{D}$ and median corneal astigmatism value was $1.13 \mathrm{D}$; both values were lowest in group 1. With regard to binocular function, $89.6 \%$ of the subjects in group 1 had 60 arcseconds or better in near stereopsis and $98.8 \%$ had no detectable strabismus, which were significantly different from the findings in the other groups. The percentage of subjects in group 1 who had 80 arcseconds or worse in near stereopsis was $10.4 \%$. In contrast, $90.9 \%$ of the subjects in group 4 had 80 arcseconds or worse in near stereopsis, and $18.2 \%$ had intermittent or manifest strabismus.

Conclusion: We suggest that examination of refraction and stereopsis in preschool-age children undergoing vision screening is an important supplement to visual acuity testing.

Keywords: vision screening, preschool children, refraction, binocular function, non-cycloplegic, hand held autorefractor

\section{Introduction}

Amblyopia is a condition that emerges when a child's visual system does not develop properly, resulting in abnormal vision in 1 or both eyes. ${ }^{1,2}$ Anisometropic, strabismic, meridional, and ametropic amblyopia are types of amblyopia commonly found in the population. Anisometropic amblyopia does not involve poor binocular vision and is the type most difficult to diagnose. It is commonly believed that the optimal time to correct amblyopia is during infancy or early childhood, before the eyes and the entire visual system, including the regions of the brain involved in vision, have fully matured. ${ }^{3,4}$ If necessary, children with refractive errors (myopia, hyperopia, or astigmatism) can wear glasses or contact lenses. However, when amblyopia does not improve with refractive correction using glasses, occlusion therapy may be considered. ${ }^{5,6}$ Although most eyecare professionals agree that amblyopia can be treated effectively in young children, many believe that treatment beyond a certain age is ineffective. There are limited data 
available to eye researchers with regard to treatment of this condition in children $>7$ or 8 years of age..$^{7-10}$

Vision screening to detect amblyopia at a sufficiently early age to enable successful treatment has been strongly recommended worldwide. ${ }^{11,12}$ In Japan, the vision screening program consists of 3 steps: ${ }^{13}$ questionnaires and home visual acuity (VA) testing (only for 3-year-old children), VA testing and inspection by health nurses at regional health centers, and detailed examinations by ophthalmologists. Schmidt et $\mathrm{al}^{14}$ reported the usefulness of refraction and binocular function testing in vision screening. Unfortunately, the rate of implementing refraction or binocular function testing has been low in many areas of Japan. ${ }^{13}$ We have been performing vision screening, including refraction and binocular function testing for early detection of eye disorders in children since 2008 in Otawara, Japan. In this cross-sectional study, we evaluated the relationship between uncorrected near VA (UNVA) and refraction and binocular function using our vision screening protocol in preschool-age children, and considered whether refraction and binocular function may explain the degree of the defect in UNVA.

\section{Material and methods Subjects}

In total, 760 children (age: $5.4 \pm 0.8$ years, range: $3-6$ years) who were enrolled in 4 nursery schools in Otawara, Japan, were recruited; a total of 1,520 eyes were examined. The procedures used in this study were approved by the Institutional Review Board of the International University of Health and Welfare (approval number 16-Io-60), and conformed to the tenets of the Declaration of Helsinki. Written informed consent was obtained from the guardians of each participant after providing an explanation of the purpose of the study and information on any risks and potential discomfort that could be experienced during the protocol.

\section{Protocol}

We assessed uncorrected VA (logarithm of the minimum angle of resolution $[\log M A R])$, objective refractive error, corneal power, stereopsis, and eye position. Uncorrected VA ( $\log$ MAR) was assessed for distant and near vision using the Landolt broken ring target (Single LANDOLT Test Cards, Handaya Co., Ltd, Tokyo, Japan). UNVA data were used in the analysis to reduce the possibility of diagnosing subjects with mild myopia or astigmatism requiring no corrective refraction as poor visual acuity. Objective refractive error and corneal power were measured in the natural pupil of each eye using a portable autorefractor/keratometer (Retinomax K Plus 3, Righton, Tokyo, Japan). Data with regard to spherical equivalent value, cylindrical power, corneal power, and corneal astigmatism were collected; the inter-ocular differences in spherical equivalent value (ie, anisometropia) were calculated and analyzed. Near stereopsis was measured using the Titmus stereo test ${ }^{15}$ (Stereo Optical Co., Inc., Chicago, IL, USA) at $40 \mathrm{~cm}$. The Titmus stereo test, which we selected in this study, is one of the several standard stereopsis tests in amblyopia screening and can measure stereopsis of up to 40 arcseconds. Eye position was evaluated using the cover, cover-uncover, and alternate cover tests while the children gazed at a distant point without spectacles. Eye position tests measured baseline eye position at distance to exclude the effect of accommodative and proximal convergence. Subjects were divided into 1 of 4 subgroups according to the lowest UNVA value compared between the 2 eyes: group 1 (VA $\leq$ logMAR 0.00), group 2 (VA $\log$ MAR 0.15-0.05), group 3 (VA logMAR 0.52-0.22), and group 4 (VA $>$ logMAR 0.52). These parameters were compared among the groups. This standardized grouping allocation is used for the determination of VA in vision screening in Japan.

\section{Statistical analyses}

Age, spherical equivalent value, anisometropia, cylindrical power, corneal power, and corneal astigmatism were compared among the groups using analysis of variance, namely, the Kruskal-Wallis and Steel-Dwass tests. ${ }^{16-18}$ Stereopsis was calculated as the percentage of subjects in each group as $\leq 60$ arcseconds, $\geq 80$ arcseconds, or unmeasurable. In the Titmus stereo test, when subjects failed in the animal test, and in stereopsis of 800 arcseconds in the circle test, we defined stereopsis as unmeasurable. Parks ${ }^{19}$ reported that the limitation of stereopsis for monofixation phoria was 67 arcseconds. In addition, Birch et $\mathrm{al}^{20}$ reported that mean stereo acuity at 5 years of age was 60 arcseconds. The mean age in this study was 5.4 years, and we defined 60 arcseconds as the standard value of stereopsis in the fovea of each eye. Eye position was calculated as the percentage of the subjects with no, intermittent, and manifest strabismus. These values were indicated to examine associations among the groups using the chi-squared test and Cramer's V. ${ }^{21}$ Statistical analysis was performed using SPSS version 23.0 (IBM Corp., Armonk, NY, USA), with $P<0.05$ considered to be statistically significant.

\section{Results}

The sample number of each of the 4 sub-groups was as follows: group 1, 608 children; group 2, 114 children; group 3, 27 children; and group 4, 11 children. 
Table I Details of the subject's parameters

\begin{tabular}{|c|c|c|c|c|c|}
\hline Groups & Group I & Group 2 & Group 3 & Group 4 & $P$-value \\
\hline Sample number & 608 & 114 & 27 & 11 & - \\
\hline Age (years) & $5.5 I \pm 0.68(6.00)$ & $5.11 \pm 0.88(5.00)$ & $4.96 \pm 1.09(5.00)$ & $5.00 \pm 0.77(5.00)$ & $<0.0001$ \\
\hline Uncorrected distant visual acuity (logMAR) & $-0.03 \pm 0.04(0.00)$ & $0.04 \pm 0.08(0.00)$ & $0.27 \pm 0.24(0.22)$ & $0.56 \pm 0.29(0.52)$ & - \\
\hline Uncorrected near visual acuity (logMAR) & 0.00 & $0.08 \pm 0.04(0.05)$ & $0.30 \pm 0.11(0.30)$ & $0.84 \pm 0.24(0.76)$ & - \\
\hline Spherical equivalent value (D) & $-1.39 \pm 1.28(-1.13)$ & $-1.17 \pm 1.45(-1.00)$ & $-1.35 \pm 1.75(-0.88)$ & $0.91 \pm 3.08(0.13)$ & $<0.01$ \\
\hline Cylindrical power (D) & $0.40 \pm 0.37(0.25)$ & $0.69 \pm 0.53(0.50)$ & $1.66 \pm 1.10(1.50)$ & $1.55 \pm 1.07(1.00)$ & $<0.0001$ \\
\hline Anisometropia (D) & $0.57 \pm 0.55(0.38)$ & $0.73 \pm 0.68(0.56)$ & $0.94 \pm 0.80(0.75)$ & $1.60 \pm 1.58(0.86)$ & $<0.01$ \\
\hline Corneal power (D) & $43.62 \pm 1.40(43.63)$ & $43.8 I \pm 1.43(43.75)$ & $43.78 \pm 1.85(43.94)$ & $43.32 \pm 2.20(43.19)$ & 0.64 \\
\hline Corneal astigmatism (D) & $1.25 \pm 0.66(1.13)$ & $1.58 \pm 0.8 \mid(1.50)$ & $2.20 \pm 1.07(2.00)$ & $2.33 \pm 1.22(2.38)$ & $<0.0001$ \\
\hline Stereopsis $(\%,[n])$ & & & & & $<0.0001$ \\
\hline$\leq 60$ arcseconds & $89.64(545)$ & $70.17(80)$ & $44.44(12)$ & $9.09(1)$ & \\
\hline$\geq 80$ arcseconds & $8.88(54)$ & $22.81(26)$ & $44.44(12)$ & $54.55(6)$ & \\
\hline Unmeasurable & $\mathrm{I} .48(9)$ & $7.02(8)$ & $11.12(3)$ & $36.36(4)$ & \\
\hline Eye position (\%, [n]) & & & & & $<0.01$ \\
\hline No strabismus & $98.85(601)$ & $96.46(109)$ & $100.00(27)$ & $81.82(9)$ & \\
\hline Intermittent strabismus & $0.49(3)$ & $1.77(2)$ & $0.00(0)$ & $9.09(\mathrm{I})$ & \\
\hline Manifest strabismus & $0.66(4)$ & $1.77(2)$ & $0.00(0)$ & $9.09(1)$ & \\
\hline
\end{tabular}

Notes: Values of age, visual acuity and diopter are shown as mean \pm SD (median). Percentages are shown as percent (number). P-values were determined using KruskalWallis test (age and refraction) and Chi-squared test (stereopsis and eye position). Group I (VA $\leq 0.00$ [logMAR]), group 2 (VA 0.15 to 0.05 ), group 3 (VA 0.52 to 0.22 ), and group 4 (VA > 0.52).

Abbreviations: $D$, diopter; logMAR, logarithm of the minimum angle of resolution.

Table 1 summarizes the demographic information and details of the study parameters. The median age of the children in group 1 was 6 years, which was the oldest among the groups $(P<0.05$; Figure 1$)$. The median spherical equivalent values were -1.13 diopter (D) in group 1 and -1.00 in group 2 , which were more myopic than group 4 $(P<0.05$; Figure $2 \mathrm{~A})$. Median cylindrical power in group 1 was $0.25 \mathrm{D}$, and was the lowest among all groups $(P<0.05$; Figure 2B). Median anisometropia in group 1 was $0.38 \mathrm{D}$, which was lower than in the other groups $(P<0.05 ;$ Fig $2 \mathrm{C})$. Median corneal power in group 1 was $43.63 \mathrm{D}$, and was not significantly different from the other groups $(P>0.05$; Figure 2D). The median corneal astigmatism value in group 1 was $1.13 \mathrm{D}$, and was the lowest among all groups $(P<0.05$; Figure 2E).

With regard to binocular function, UNVA had a significant association with stereopsis $(P<0.05$, Cramer's $\mathrm{V}=0.45)$ and with eye position $(P<0.05$, Cramer's V $=0.12)$ (Figure 3A and B). Most (89.6\%) subjects in group 1 had 60 arcseconds or better in near stereopsis, and $98.8 \%$ had no detectable strabismus. The percentage of subjects in group 1 who had 80 arcseconds or worse in near stereopsis was $10.4 \%$. In contrast, $90.9 \%$ of the subjects in group 4 had 80 arcseconds or worse in near stereopsis and $18.2 \%$ had intermittent or manifest strabismus. Details of the eye position in each group are shown in Table 2. We also found that more subjects with strabismus had exotropia than esotropia in this study population.

\section{Discussion}

This study assessed visual function in preschool-age children using a vision screening protocol. Parameters of visual function were compared among 4 groups classified according to UNVA data.

Previous investigators have reported that the visually sensitive period in humans is up to $6-8$ years of age, ${ }^{7-10,22,23}$ and, furthermore, that accurate examination of VA is not possible in children younger than this age range. ${ }^{24} \mathrm{In}$ this study, however, we recruited 760 children between 3 and 6 years of age and, therefore, visual function may still have

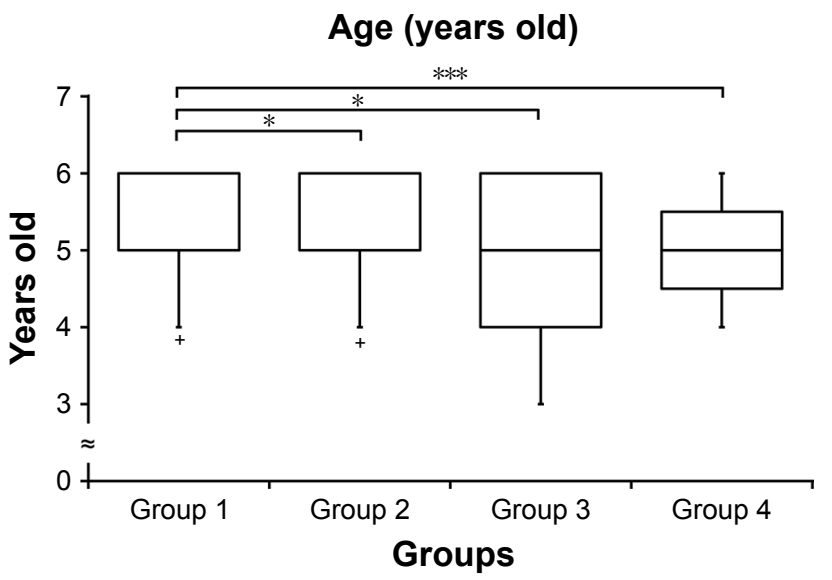

Figure I Comparison of age among groups.

Notes: Boxplot denote $5 \%, 25 \%, 50 \%, 75 \%$, and $95 \%$ intervals, and cross marks denote outliers. *Shows $P$-values determined using a Steel-Dwass test. $* P<0.05$, $* * * P<0.000$ I. The median age of the children in Group I was the oldest among the groups. Group I (VA $\leq 0.00$ [logMAR]), group 2 (VA 0.15 to 0.05 ), group 3 (VA 0.52 to 0.22 ), and group 4 (VA $>0.52$ ). 


\section{A Spherical equivalent value (D)}

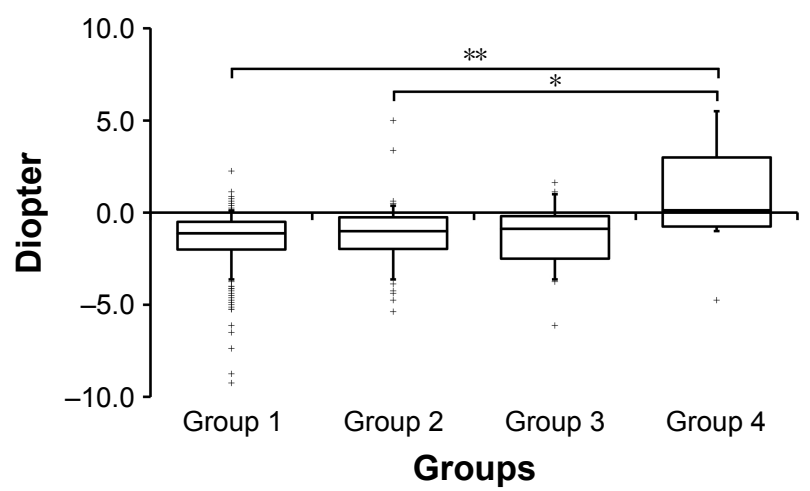

C

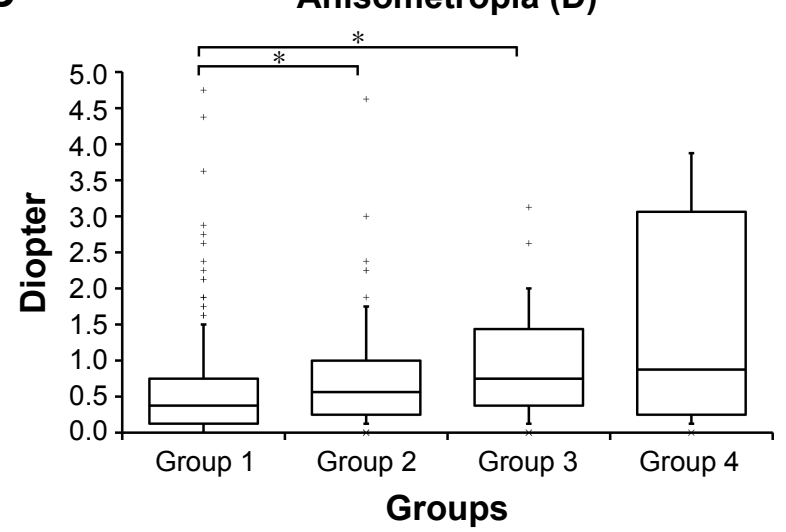

B

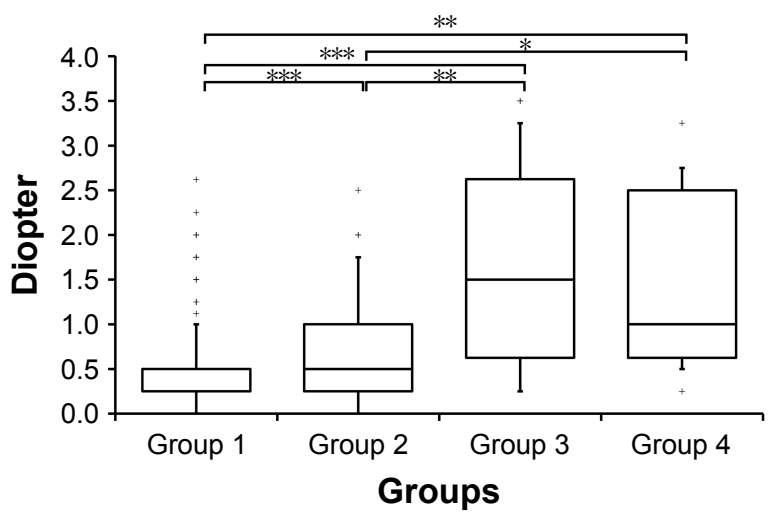

D

Corneal power (D)

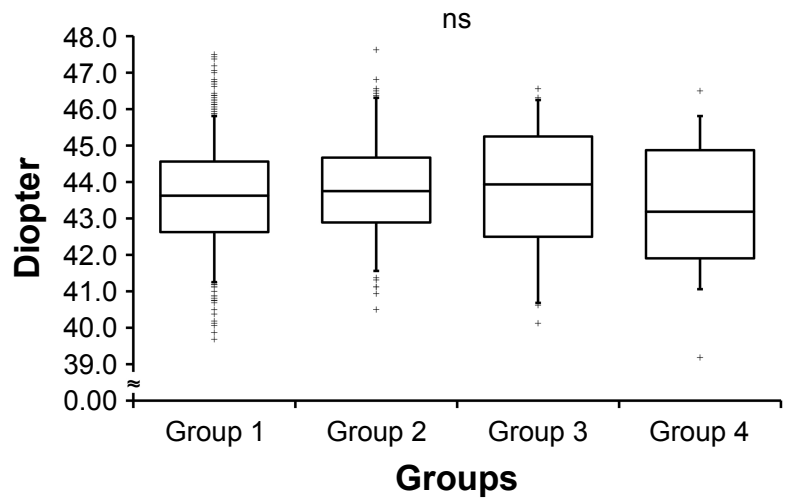

\section{E Corneal astigmatism (D)}

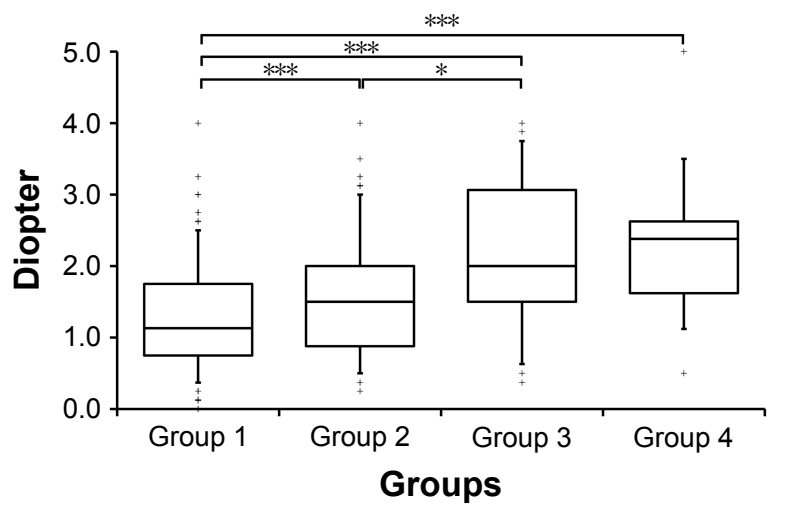

Figure 2 Comparison of refraction among groups.

Notes: (A) Spherical equivalent value. (B) Cylindrical power. (C) Anisometropia. (D) Corneal power. (E) Corneal astigmatism. Boxplot denote 5\%, 25\%, 50\%, 75\%, and $95 \%$ intervals, and cross marks denote outliers. $*$ Shows $P$-values determined using a Steel-Dwass test. $* P<0.05$, $* * P<0.0$ I, $* * * P<0.000$ I. The median spherical equivalent values in Group I and 2 were significantly more myopic than in Group $4(P<0.05)$. Median cylindrical power in Group I was the lowest among all groups $(P<0.05)$. Median anisometropia in Group I was significantly lower than in Groups 2 and $3(P<0.05)$. Median corneal power was not significantly different among groups $(P>0.05)$. The median corneal astigmatism value in Group I was the lowest among all groups $(P<0.05)$. Group I (VA $\leq 0.00$ [logMAR]), group 2 (VA 0.15 to 0.05 ), group 3 (VA 0.52 to 0.22 ), and group 4 (VA > 0.52).

Abbreviation: ns, not significant.

been in the early stages of development. The median age of children in group 1 (ie, VA $\leq \log$ MAR 0.00) was greater than in the other groups.

According to objective measures of refractive error using the Retinomax system, the mean value of refractive error shift to myopia was approximately $-1.00 \mathrm{D} .{ }^{25}$ The median (mean) spherical equivalent value was $-1.13(-1.39) \mathrm{D}$ in group 1 .
This result was obtained by measurement without cycloplegia, and the median (mean) spherical equivalent value with cycloplegia in groups 1, 2, and 3 may have moved closer to emmetropia. Since group 4 showed significant hypermetropia, most defects in VA in group 4 may have been caused by amblyopia due to hypermetropic anisometropia or hypermetropia. The number of refractive errors (eg, cylindrical 
A

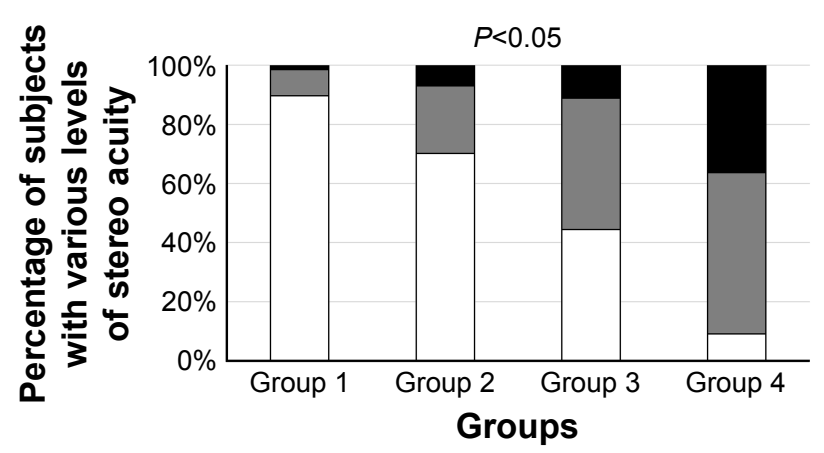

$\square \leq 60$ arcseconds
$\square \geq 80$ arcseconds
$\square$ Unmeasurable
B

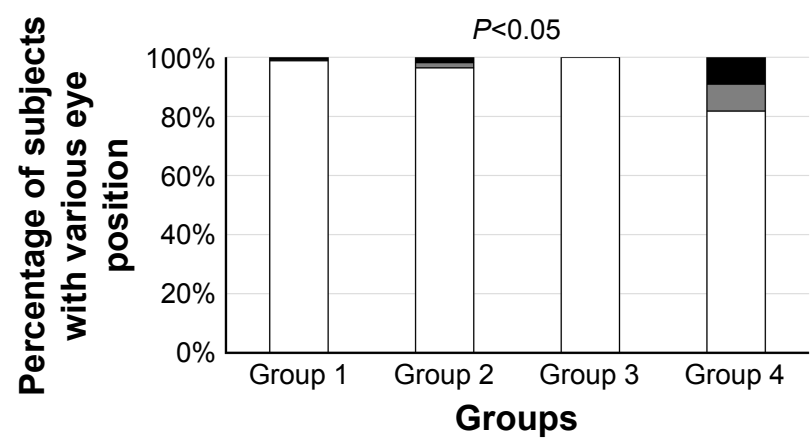

$\square$ No strabismus

$\square$ Intermittent strabismus

- Manifest strabismus

Figure 3 Comparison of binocular function among groups.

Notes: (A) Stereopsis. Bar graph with $100 \%$ stacked columns. The white bar shows the percentage of children with $\leq 60$ arcseconds, and the gray bar shows the percentage of children with $\geq 80$ arcseconds; the black bar signifies that the value was unmeasurable. (B) Eye position. Bar graph with I00\% stacked columns. The white bar shows the percentage of children with no strabismus, and the gray bar shows the percentage of children with intermittent strabismus; the black bar signifies manifest strabismus. Uncorrected near visual acuity was significantly associated with stereopsis $(P<0.05$, Cramer's $\vee=0.45)$ and eye position $(P<0.05$, Cramer's $\vee=0.12)$. Group I (VA $\leq 0.00$ [logMAR]), group 2 (VA 0.15 to 0.05 ), group 3 (VA 0.52 to 0.22 ), and group 4 (VA $>0.52$ ).

power, anisometropia, and corneal astigmatism) in children in group 1 was the lowest among all groups. There was a trend showing that the extent of these refractive errors was related to UNVA; therefore, examination of manifest refraction may serve as a rough estimation of VA.

In analyzing stereopsis, the percentage of subjects who had 60 arcseconds or better was lower in the poorer UNVA groups. Additionally, our study revealed similar tendencies to those described in previous reports, in which stereopsis correlated with VA. ${ }^{26-28}$ The percentage of subjects in group 1 who had 80 arcseconds or worse in near stereopsis was $8.9 \%$. Thus, we can suspect VA defects solely by performing stereopsis testing when the subjects' stereopsis is 80 arcseconds or worse. In contrast, eye position correlated poorly with VA defects. In group 4, 18.2\% of subjects had intermittent or manifest strabismus. Schmidt et $\mathrm{al}^{14}$ and Ciner et $\mathrm{al}^{28}$ reported that the sensitivity of the stereopsis test for detecting children with amblyopia was 0.63 and of the cover test was 0.27 , and also that these sensitivities increased with increasing severity of vision disorders. Our results showed a similar tendency to that reported by previous studies. Therefore, we suggest that examination of binocular function for preschool-aged children undergoing vision screening is an important supplement in VA testing and the detection of strabismus. In addition, it was reported that the proportion of children who could not be tested was $9.7 \%$ and $2.1 \%$ in the stereopsis and cover tests, respectively. ${ }^{14}$ When VA and stereopsis tests fail due to low compliance of children, qualitative testing using the cover and cover-uncover tests may be a useful index for detecting suspected VA defects.

The study was limited in that the best-corrected VA and the refractive error with cycloplegia were not taken into consideration due to screening constraints. The sensitivity and specificity of testing in this protocol could be determined with the addition of gold standard examinations for providing a complete ocular assessment of the child after this protocol is implemented, as has been shown in previous reports. ${ }^{14,29}$ We believe that future studies that will include the results

Table 2 Details of the eye position in each group

\begin{tabular}{|c|c|c|c|c|}
\hline Groups & Group I & Group 2 & Group 3 & Group 4 \\
\hline Number, n (\%) & $608(100)$ & $114(100)$ & $27(100)$ & II (I00) \\
\hline Number of no-shift, n (\%) & $423(69.5)$ & $73(64.0)$ & $18(66.7)$ & $7(63.6)$ \\
\hline Number of exophoria, n (\%) & $174(28.6)$ & $36(31.5)$ & $9(33.3)$ & $2(18.2)$ \\
\hline Number of intermittent exotropia, n (\%) & $3(0.5)$ & $2(1.8)$ & $0(0.0)$ & I (9.I) \\
\hline Number of exotropia, n (\%) & $3(0.5)$ & I (0.9) & $0(0.0)$ & I (9.I) \\
\hline Number of esophoria, n (\%) & $4(0.7)$ & $0(0.0)$ & $0(0.0)$ & $0(0.0)$ \\
\hline Number of intermittent esotropia, n (\%) & $0(0.0)$ & $0(0.0)$ & $0(0.0)$ & $0(0.0)$ \\
\hline Number of esotropia, n (\%) & I (0.2) & I (0.9) & $0(0.0)$ & $0(0.0)$ \\
\hline Number of unmeasurable, n (\%) & $0(0.0)$ & I (0.9) & $0(0.0)$ & $0(0.0)$ \\
\hline
\end{tabular}

Notes: Numbers are shown as number (percent). Group I (VA $\leq 0.00$ [logMAR]), group 2 (VA 0.15 to 0.05 ), group 3 (VA 0.52 to 0.22 ), and group 4 (VA $>0.52$ ). 
of detailed examinations by eyecare professionals (eg, ophthalmologists, optometrists, and orthoptists) after vision screening are necessary (ie, follow-up studies).

\section{Conclusion}

In this study, relationships of refractive error, corneal power, stereopsis, and eye position with UNVA were evaluated. Therefore, in addition to VA testing, we suggest that binocular function tests warrant consideration in vision screening protocols.

\section{Acknowledgment}

This work was supported by JSPS KAKENHI Grant Number 17K13249, 16K21322 and 15H03515.

\section{Author contributions}

All authors contributed toward data analysis, drafting and revising the paper and agree to be accountable for all aspects of the work.

\section{Disclosure}

The authors report no conflicts of interest in this work.

\section{References}

1. Awaya S, Miyake S. Form vision deprivation amblyopia: further observations. Graefes Arch Clin Exp Ophthalmol. 1988;226(2):132-136.

2. Awaya S, Watanabe Y. Amblyopia. Curr Opin Ophthalmol. 1995; 6(5):9-14.

3. Von Noorden GK. [Treatment of amblyopia]. Fortschr Ophthalmol. 1990;87 Suppl:149-154. German.

4. Gregg FM, Parks MM. Stereopsis after congenital monocular cataract extraction. Am J Ophthalmol. 1992;114(3):314-317.

5. Holmes JM, Beck RW, Kraker RT, et al; Pediatric Eye Disease Investigator Group. Impact of patching and atropine treatment on the child and family in the amblyopia treatment study. Arch Ophthalmol. 2003;121(11): $1625-1632$

6. Cotter SA; Pediatric Eye Disease Investigator Group, Edwards AR, et al. Treatment of anisometropic amblyopia in children with refractive correction. Ophthalmology. 2006;113(6):895-903.

7. Kuman IG, Fedorov SN, Novikova LA. [The sensitive period in the development of the human visual system]. Zh Vyssh Nerv Deiat Im I P Pavlova. 1983;33(3):434-441. Russian.

8. Awaya S, Sugawara M, Miyake S. Observations in patients with occlusion amblyopia: results of treatment. Trans Ophthalmol Soc U K. 1979; 99(3):447-454.

9. Epelbaum M, Milleret C, Buisseret P, Dufier JL. The sensitive period for strabismic amblyopia in humans. Ophthalmology. 1993;100(3): 323-327.

Clinical Ophthalmology

\section{Publish your work in this journal}

Clinical Ophthalmology is an international, peer-reviewed journal covering all subspecialties within ophthalmology. Key topics include: Optometry; Visual science; Pharmacology and drug therapy in eye diseases; Basic Sciences; Primary and Secondary eye care; Patient Safety and Quality of Care Improvements. This journal is indexed on Submit your manuscript here: http://www.dovepress.com/clinical-ophthalmology-journal
10. Hardman Lea SJ, Loades J, Rubinstein MP. The sensitive period for anisometropic amblyopia. Eye (Lond). 1989;3(Pt 6):783-790.

11. Ciner EB, Schmidt PP, Orel-Bixler D, et al. Vision screening of preschool children: evaluating the past, looking toward the future. Optom Vis Sci. 1998;75(8):571-584.

12. Eibschitz-Tsimhoni M, Friedman T, Naor J, Eibschitz N, Friedman Z. Early screening for amblyogenic risk factors lowers the prevalence and severity of amblyopia. J AAPOS. 2000;4(4):194-199.

13. Yamada M. [Screening programs for amblyopia in children]. $J$ Eye. 2010;27:1635-1639. Japanese.

14. Schmidt P, Maguire M, Dobson V, et al; Vision in Preschoolers Study Group. Comparison of preschool vision screening tests as administered by licensed eye care professionals in the Vision in Preschoolers Study. Ophthalmology. 2004;111(4):637-650.

15. Simons K, Reinecke RD. A reconsideration of amblyopia screening and stereopsis. Am J Ophthalmol. 1974;78(4):707-713.

16. Kruskal WH, Wallis WA. Use of ranks in one-criterion variance analysis. J Am Stat Assoc. 1952;47(260):583-621.

17. Steel RGD. A rank sum test for comparing all pairs of treatments. Technometrics. 1960;2(2):197-207.

18. Dwass M. Some k-sample rank-order tests. In: Olkin I, Ghurye SG, Hoeffding H, Madow WG, Mann HB, editors. Contributions to Probability and Statistics. Stanford, CA, USA: Stanford University Press; 1960: 198-202.

19. Parks M. Stereoacuity as an indicator of bifixation. International Strabismus Symposium. Basel, Switzerland: Karger; 1968:258.

20. Birch E1, Williams C, Drover J, et al. Randot Preschool Stereoacuity Test: normative data and validity. J AAPOS. 2008;12(1):23-26.

21. Cramer H. Mathematical Methods of Statistics. Princeton: Princeton University Press; 1946.

22. Magoon EH, Robb RM. Development of myelin in human optic nerve and tract. A light and electron microscopic study. Arch Ophthalmol. 1981; 99(4):655-659.

23. Teller DY. Measurement of visual acuity in human and monkey infants: the interface between laboratory and clinic. Behav Brain Res. 1983; 10(1):15-23.

24. Shute R, Candy R, Westall C, Woodhouse JM. Success rates in testing monocular acuity and stereopsis in infants and young children. Ophthalmic Physiol Opt. 1990;10(2):133-136.

25. Choong YF, Chen AH, Goh PP. A comparison of autorefraction and subjective refraction with and without cycloplegia in primary school children. Am J Ophthalmol. 2006;142(1):68-74.

26. Lee SY, Isenberg SJ. The relationship between stereopsis and visual acuity after occlusion therapy for amblyopia. Ophthalmology. 2003;110(11): 2088-2092.

27. Pediatric Eye Disease Investigator Group. Clinical factors associated with moderate hyperopia in preschool children with normal stereopsis and visual acuity. $J$ AAPOS. 2016;20(5):455-457.

28. Ciner EB, Ying GS, Kulp MT, et al; Vision in Preschoolers Study Group. Stereoacuity of preschool children with and without vision disorders. Optom Vis Sci. 2014;91(3):351-358.

29. Hendler K, Mehravaran S, Lu X, Brown SI, Mondino BJ, Coleman AL. Refractive errors and Amblyopia in the UCLA Preschool Vision Program; first year results. Am J Ophthalmol. 2016;172:80-86.

\section{Dovepress}

PubMed Central and CAS, and is the official journal of The Society of Clinical Ophthalmology (SCO). The manuscript management system is completely online and includes a very quick and fair peer-review system, which is all easy to use. Visit http://www.dovepress.com/ testimonials.php to read real quotes from published authors. 